\title{
Detailed numerical modeling of a linear parallel-plate Active Magnetic Regenerator
}

Nielsen, Kaspar Kirstein; Bahl, Christian Robert Haffenden; Smith, Anders; Bjørk, Rasmus; Pryds, Nini; Hattel, Jesper Henri

Published in:

International Journal of Refrigeration

Link to article, DOI:

10.1016/j.jirefrig.2009.03.003

Publication date:

2009

Link back to DTU Orbit

Citation (APA):

Nielsen, K. K., Bahl, C. R. H., Smith, A., Bjørk, R., Pryds, N., \& Hattel, J. H. (2009). Detailed numerical modeling of a linear parallel-plate Active Magnetic Regenerator. International Journal of Refrigeration, 32(6), 1478-1486. https://doi.org/10.1016/j.jijefrig.2009.03.003

\section{General rights}

Copyright and moral rights for the publications made accessible in the public portal are retained by the authors and/or other copyright owners and it is a condition of accessing publications that users recognise and abide by the legal requirements associated with these rights.

- Users may download and print one copy of any publication from the public portal for the purpose of private study or research.

- You may not further distribute the material or use it for any profit-making activity or commercial gain

- You may freely distribute the URL identifying the publication in the public portal 


\title{
Detailed numerical modeling of a linear parallel-plate Active Magnetic Regenerator
}

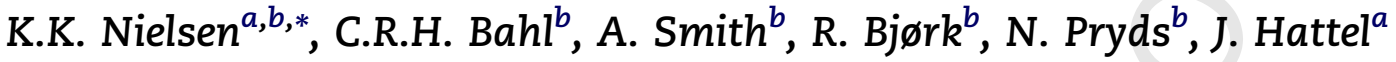 \\ ${ }^{a}$ Department of Mechanical Engineering, Technical University of Denmark, Building 425, Niels Koppels Allé, DK-2800 Kgs. Lyngby, Denmark \\ ${ }^{\mathrm{b}}$ Fuel Cells and Solid State Chemistry Division, Risø National Laboratory for Sustainable Energy, Technical University of Denmark, \\ Frederiksborguej 399, DK-4000, Denmark
}

\section{A R T I C L E I N F O}

Article history:

Received 10 December 2008

Received in revised form

2 March 2009

Accepted 16 March 2009

Published online

Keywords:

Magnetic refrigerator

Modeling

Magnetic field

Gadolinium

Heat loss

\begin{abstract}
A B S T R A C T
A numerical model simulating Active Magnetic Regeneration (AMR) is presented and compared to a selection of experiments. The model is an extension and re-implementation of a previous two-dimensional model. The new model is extended to $2.5 \mathrm{D}$, meaning that parasitic thermal losses are included in the spatially not-resolved direction.

The implementation of the magnetocaloric effect (MCE) is made possible through a source term in the heat equation for the magnetocaloric material (MCM). This adds the possibility to model a continuously varying magnetic field.

The adiabatic temperature change of the used gadolinium has been measured and is used as an alternative MCE than mean field modeling. The results show that using the 2.5D formulation brings the model significantly closer to the experiment. Good agreement between the experimental results and the modeling was obtained when using the 2.5D formulation in combination with the measured adiabatic temperature change.
\end{abstract}

(c) 2008 Elsevier Ltd and IIR.

of permanent magnet assemblies, focused research into relevant magnetocaloric materials and system/regenerator designs (e.g. Bjørk et al., 2008; Pecharsky and Gschneidner, 2006; Rowe and Barclay, 2003; Rowe and Tura, 2008).

Magnetic refrigeration at room temperature is a topic that spans several research areas. These include the optimal design

Abbreviations: AMR, Active Magnetic Regeneration; MCE, Magnetocaloric effect; MCM, Magnetocaloric material; MFT, Mean field theory; HHEX, Hot heat exchanger; CHEX, Cold heat exchanger; PDE, Partial Differential Equation; FEM, Finite Element Method; ADI, Alternate Direction Implicit; TDMA, Tri-diagonal Matrix Algorithm; Gd, Gadolinium.

* Corresponding author: +45 46774758.

E-mail address: kaspar.kirstein.nielsen@risoe.dk (K.K. Nielsen). 0140-7007/\$ - see front matter (c) 2008 Elsevier Ltd and IIR. doi:10.1016/j.ijrefrig.2009.03.003 


\begin{tabular}{|c|c|}
\hline \multicolumn{2}{|c|}{ Nomenclature } \\
\hline \multicolumn{2}{|c|}{ Variables } \\
\hline$\Delta \mathrm{T}_{\mathrm{ad}}$ & Adiabatic temperature change $[\mathrm{K}]$ \\
\hline $\mathrm{T}$ & Temperature $[\mathrm{K}]$ \\
\hline$T_{\infty}$ & Ambient temperature $[\mathrm{K}]$ \\
\hline$c_{\mathrm{p}}$ & Specific heat capacity $[J / \mathrm{kg} \mathrm{K}]$ \\
\hline$\rho$ & Mass density $\left[\mathrm{kg} / \mathrm{m}^{3}\right]$ \\
\hline$k$ & Thermal conductivity [W/m K] \\
\hline$h$ & Convective heat transfer coefficient $\left[\mathrm{W} / \mathrm{m}^{2} \mathrm{~K}\right]$ \\
\hline t & Time $[\mathrm{s}]$ \\
\hline$\Delta t$ & Timestep [s] \\
\hline$\tau_{1}$ & Timing of magnetization part of the AMR cycle [s \\
\hline$\tau_{2}$ & Timing of hot blow part of the AMR cycle [s] \\
\hline$\tau_{3}$ & Timing of demagnetization part of the AMR cycle [s \\
\hline$\tau_{4}$ & Timing of cold blow part of the AMR cycle [s] \\
\hline$\tau_{\text {rel }}$ & Equal to $\tau_{1} / \tau_{2}=\tau_{3} / \tau_{4}[-]$ \\
\hline$\tau_{\text {tot }}$ & Equal to $2\left(\tau_{1}+\tau_{2}\right)[\mathrm{s}]$ \\
\hline$\mu_{0}$ & Vacuum permeability equal to $4 \pi 10^{-7}\left(\mathrm{~N} / \mathrm{A}^{2}\right)$ \\
\hline$\mu_{0} \mathrm{H}$ & Magnetic field $[\mathrm{T}]$ \\
\hline B & Magnetic flux density [T] \\
\hline$m$ & Magnetization $\left[\mathrm{Am}^{2} / \mathrm{kg}\right]$ \\
\hline u & Velocity vector $[\mathrm{m} / \mathrm{s}]$ \\
\hline$u$ & $x$-direction velocity component $[\mathrm{m} / \mathrm{s}]$ \\
\hline$v$ & $y$-direction velocity component $[\mathrm{m} / \mathrm{s}]$ \\
\hline$\tilde{u}$ & Inlet fluid velocity $[\mathrm{m} / \mathrm{s}]$ \\
\hline
\end{tabular}

The theoretical advantages of magnetic refrigeration compared to conventional gas vaporization based refrigeration are: significantly higher energy efficiency, low-noise operation and non-toxic magnetocaloric materials and heat transfer fluids.

So far numerous experiments have been done that are based on the Active Magnetic Regenerator (AMR) cycle (e.g. Rowe et al., 2004; Gao et al., 2006; Okamura et al., 2006; Bahl et al., 2008). These experiments show in general that it is certainly possible to utilize the magnetocaloric effect (MCE), which manifests itself as an adiabatic temperature change $\left(\Delta T_{\mathrm{ad}}\right)$, inherent in the magnetocaloric material (MCM), to lift the temperature span of the AMR device to several times that of $\Delta T_{\text {ad }}$ of the used material. The experiments differ mainly in the basic design of the regenerator; some use porous packed beds and some parallel plates (of MCM). The optimal geometrical configuration of the regenerator is not obvious and since building experiments that span a sufficient number of configurations is both time-consuming and demands a great amount of resources, the need for fast and in particular accurate modeling is great.

The AMR models previously published have been onedimensional (e.g. Dikeos et al., 2006; Li et al., 2006; Engelbrecht et al., 2005; Shir et al., 2005; Allab et al., 2005) with the exception of one, published in Petersen et al. (2008), which is a two-dimensional model of a parallel-plate design. In the one-dimensional models the regenerator is discretized with a sufficient number of grid cells in the $x$-direction (parallel to the flow) and thus a lumped analysis needs to be employed in order to describe the heat transfer between the active MCM and the heat transfer fluid. This

$\begin{array}{ll}\mu & \text { Dynamic viscosity }[\mathrm{kg} / \mathrm{m} \mathrm{s}] \\ \mathrm{Re} & \text { Reynolds number }[-] \\ \mathrm{H} & \text { Height }[\mathrm{m}] \\ \mathrm{L} & \text { Regenerator length }[\mathrm{m}] \\ p & \text { Absolute pressure }\left[\mathrm{N} / \mathrm{m}^{2}\right] \\ \Delta p & \text { Pressure drop }\left[\mathrm{N} / \mathrm{m}^{2}\right] \\ \Delta \mathrm{x} & \text { Finite difference length in the } x \text {-direction }[\mathrm{m}] \\ \Delta y & \text { Finitee difference length in the } y \text {-direction }[\mathrm{m}] \\ \Delta \mathrm{z} & \text { Finite difference length in the } \mathrm{z} \text {-direction }[\mathrm{m}] \\ \mathrm{R} & \text { Thermal resistance }[\mathrm{K} / \mathrm{W}] \\ \mathrm{Q} & \text { Thermal source term }[\mathrm{W}] \\ \mathrm{Sub} \text { - and super-scripts } \\ \mathrm{f} & \text { Fluid } \\ \mathrm{s} & \text { Solid } \\ \mathrm{r} & \text { Regenerator } \\ \mathrm{m} & \text { Material (solid or fluid) } \\ \mathrm{l} & \text { Summation dummy index } \\ \mathrm{pl} & \text { Plate } \\ \mathrm{conv} & \text { Convection } \\ \mathrm{i} & \mathrm{x} \text {-direction index } \\ \mathrm{j} & \mathrm{y} \text {-direction index } \\ 0 & \text { Value at time } t \\ * & \text { Value at time } t+1 / 2 \Delta t \\ * * & \text { Value at time } t+\Delta t\end{array}$

description is the main simplification compared to a twodimensional model that also resolves the thickness of both the fluid channels and MCM-plates. The model is developed for a parallel-plate based design; it would be much more tedious to develop a consistent 2D model of a porous bedbased design.

This work presents a 2.5-dimensional model that is a further development and re-implementation of the model presented in Petersen et al. (2008). This new model was developed in order to decrease computation time, make it much more versatile in terms of geometrical and operational configurations and to include parasitic thermal losses in a physically realistic way in order to resemble the current experimental AMR device situated at Risø DTU in Denmark (see Bahl et al., 2008).

The outline of this paper is the following: In Section 2 the model is presented. In Section 3 the conditions of the experimental setup are implemented into the model. The model is validated in various geometrical and operational configurations. Finally in Section 4 the conclusions are drawn and future work is presented and proposed.

\section{The numerical model}

The model is designed to resemble a reciprocating linear parallel-plate based AMR design. The basic model is thoroughly discussed in Petersen et al. (2008). The following subsection is a short summary of that model and in Subsections 2.2-2.4 new additions are presented. 


\subsection{Summary of the original model}

Fig. 1 shows the geometry and boundary conditions in detail. The plates are stacked with an equal spacing that defines the fluid channel thickness. The plates can be made of any MCM and the heat transfer fluid can be any liquid of interest. The $x$ direction is defined to be parallel to the flow. The $y$-direction is perpendicular to the plane of the MCM-plates. The z-direction, which is not resolved in the basic model denotes the width of the fluid channel and MCM-plate. Placed at either end in the $x-$ direction are a cold and a hot heat exchanger, respectively. The model includes half a plate and half a fluid channel in the $y$-direction in a so-called replicating cell thus exploiting the symmetry of both the fluid channel and plate.

When the fluid displacement is modeled the fluid-domain is kept stationary and the solid domains (i.e. heat exchangers (HEXs) and the MCM-plate) are subject to a movement correspondingly and an appropriate fluid-flow profile is applied to the fluid-domain. The heat exchangers at either end ensure a smooth way of measuring the temperature span for a no heat-load modeling situation. In the case of a heat-load modeling situation the cold heat exchanger (CHEX) is kept at a fixed temperature via its upper boundary. The hot heat exchanger (HHEX) is at all times kept at the ambient temperature $T_{\infty}$ via its upper boundary condition.

The AMR cycle simulated includes four steps. The total cycle time is denoted by $\tau_{\text {tot }}$ and the timings of the four substeps are denoted by $\tau_{1}, \tau_{2}, \tau_{3}$ and $\tau_{4}$ respectively. The cycle is symmetric meaning that $\tau_{1}=\tau_{3}$ and $\tau_{2}=\tau_{4}$. The first step is the magnetization of the MCM. The second step is the so-called "hot blow", i.e. where the fluid is moved from the cold towards the hot end. In the third step the MCM is demagnitized. The fourth step is called the "cold blow" and during this step the fluid is moved from the hot end towards the cold end. The timing fraction of the magnetization periods to the blow periods is defined as $\tau_{\mathrm{re}} \equiv \tau_{1} / \tau_{2}=\tau_{3} / \tau_{4}$.

The MCE is modeled via mean field theory (MFT) (see Petersen et al., 2008) and the resulting $\Delta T_{\text {ad }}$ is directly applied as a discrete temperature increase/decrease in the control volume under consideration. The specific heat capacity $c_{\mathrm{p}}(\mathrm{H}$, $\mathrm{T}$ ) is also calculated (as a function of both temperature and field) from MFT and is updated in every timestep. The MCE is highly sensitive to impurities and variations in the MCM, which are not modeled by MFT. It is therefore imperative that experimental data are used when available.

The equation system solved consists of four partial differential equations (PDEs) coupled via inner boundaries. For the solid domains (subscript s) the equations are all unsteady diffusion equations (for convenience subscript $s$ has been adopted for all three solid domains, though the material properties $\rho, k$ and $c_{\mathrm{p}}$ are not the same):

$$
\rho_{\mathrm{s}} c_{\mathrm{p}, \mathrm{s}} \frac{\partial \mathrm{T}_{\mathrm{s}}}{\partial \mathrm{t}}=k_{\mathrm{s}} \nabla^{2} \mathrm{~T}_{\mathrm{s}} .
$$

Here, the mass density is $\rho$, the temperature is $T$, time is $t$ and the thermal conductivity is $k$. The PDE describing the transient thermal behaviour of the fluid-domain, subscript $f$, includes an extra term, namely the convective heat transfer:

$\rho_{\mathrm{f}} c_{\mathrm{p}, \mathrm{f}}\left(\frac{\partial T_{\mathrm{f}}}{\partial \mathrm{t}}+(u \cdot \nabla) \mathrm{T}_{\mathrm{f}}\right)=k_{\mathrm{f}} \nabla^{2} T_{\mathrm{f}}$.

The fluid velocity is denoted by $u=(u, v)$. Thus, all the thermal properties except $c_{\mathrm{p}}$ for the MCM are assumed constant.

\subsubsection{Velocity profile}

The applied velocity field is a steady, incompressible, fully developed and laminar flow de-coupled from the thermal system. The boundary conditions are non-slip on the boundary between the fluid-domain and the solid domains and slip on the symmetry boundary.

The assumption of incompressible flow is certainly valid since water (or a water + ethanol mixture) is used as the heat transfer fluid. Since the thermal properties $\left(\rho, c_{\mathrm{p}}\right.$ and $\left.k\right)$ of water do not change significantly under the present working conditions, these are safely assumed to be constant and thus de-coupled from the thermal system.

The Reynolds number of the system is given by

$\operatorname{Re}=\frac{2 \mathrm{H}_{\mathrm{f}} \tilde{u} \rho_{\mathrm{f}}}{\mu}$,

where $\tilde{u}$ is the inlet velocity, $H_{\mathrm{f}}$ the fluid channel thickness and $\mu$ the dynamic viscosity of the fluid. The mass density and viscosity are constant $\left(\rho_{\mathrm{f}}=997 \mathrm{~kg} / \mathrm{m}^{3}\right.$ and $\mu=8.91 \times 10^{-4} \mathrm{~kg} /$ $\mathrm{m} \mathrm{s}$ ) and the most extreme (i.e. in this case maximum) values of $\tilde{u}$ and $H_{\mathrm{f}}$ are $0.01 \mathrm{~m} / \mathrm{s}$ and $0.8 \times 10^{-3} \mathrm{~m}$, respectively. This yields a maximum value of the Reynolds number to be $\approx 24$. This value is well within the range of laminar flows justifying our assumption.

Balancing the convective and viscous terms in the incompressible Navier-Stokes equations the entrance length, $L$, of a laminar pipe-flow can be found to be $L=0.06 \mathrm{H}_{\mathrm{f}} R$ e (e.g. Lautrup, 2005). For the extreme case where $\tilde{u}$ and $H_{\mathrm{f}}$ attain their maximum values the entrance length is about $0.001 \mathrm{~m}$ and thus compared to the length of the flow channel $\left(L_{f}=0.16 \mathrm{~m}\right)$ the assumption of fully developed flow is valid.

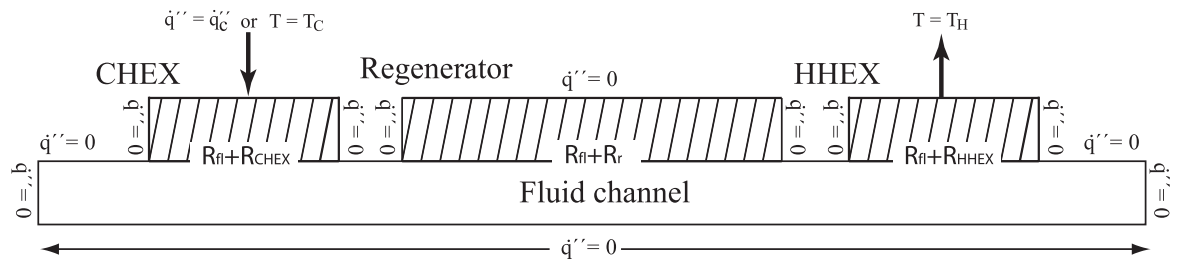

Fig. 1 - Two-dimensional slice of the original model. Half a fluid channel, MCM-plate and HEXs are seen. The thermal boundary conditions are indicated. 
The only non-zero velocity component is the $x$-direction velocity $u$. The steady incompressible Navier-Stokes equations for laminar flows can thus be reduced to

$\mu \frac{\partial^{2} u}{\partial y^{2}}=\frac{\partial p}{\partial x}$

where the pressure gradient in the flow-direction is given by $\partial p / \partial x=\Delta p / L_{\mathrm{f}}$. We assume the pressure drop to be constant, given by $\Delta p=12 \mathrm{~L}_{\mathrm{f}} \mu \tilde{\mathrm{u}} / \mathrm{H}_{\mathrm{f}}^{2}$ (Fox and McDonald, 1994). Integration of Eq. (4) and utilization of the boundary conditions $u\left(y=1 / 2 H_{f}\right)=\tilde{u}$ and $\partial U /\left.\partial y\right|_{y=0}=0$, where $y=0$ is defined as the middle of the flow channel and $y=1 / 2 \mathrm{H}_{\mathrm{f}}$ is the upper boundary between the fluid channel and solid domain, gives the well-known velocity profile

$$
u(y)=\tilde{u}\left(\frac{6 y^{2}}{H_{f}^{2}}-1 / 2\right)
$$

\subsection{The numerical scheme}

In the original model Eqs. (1) and (2) were solved using the commercial software package Comsol (Comsol, 2005). The numerical discretization was based on the Finite Element Method (FEM) and the temporal integration was done fully implicit. The current model has been re-written using finite differences of second order and the Alternate Direction Implicit(ADI) temporal integration method. The code is written by the authors and is currently available in generic Fortran.

The reason for choosing finite differences is that total energy conservation across boundaries is guaranteed at all times due to the nature of that formalism as opposed to the FEM where the conservation of energy has to rely on interpolation methods between node points. The reason why strict energy conservation is crucial in this work is the nature of the moving boundaries. It is very important that the thermal energy exchange between the subdomains is fully conserved at all times. This can be achieved by the FEM (see Petersen et al., 2008). But the cost is a large computational time. The original Comsol model uses around $50 \mathrm{~h}$ to complete a simulation of 600 AMR cycles whereas this new code uses around $30 \mathrm{~min}$, in both cases on an Intel Core 2 Duo $2.0 \mathrm{GHz}$ Windows-based PC.

The reason for using the ADI method (see e.g. Patankar, 1980 ) is that the benefit from the implicit solution of each timestep is achieved and the speed of the explicit integration method is almost reached. The ADI scheme for two-dimensional problems is split into two sub-timesteps. In the first sub-timestep one direction is determined implicitly and the other is used explicitly. In the second sub-timestep the situation is reversed. The term "explicitly used" means that the variable solved for (e.g. temperature $T$ ) is known at the beginning of the timestep. Likewise, the term "implicitly determined" means that the variable is solved for at the new point in time.

If the index-pair $(i, j)$ defines the position in the $x$ - and $y$ direction and $T_{i, j}^{0}, T_{i, j}^{*}$ and $T_{i, j}^{* *}$ are chosen to denote the temperatures at times $t, t+1 / 2 \Delta t$ and $t+\Delta t$, respectively, for the grid cell centered at $(i, j)$ the unsteady discretized equation for the thermal conduction becomes:

$$
\begin{aligned}
\rho c_{p} \Delta x \Delta y \Delta z \frac{T_{i, j}^{*}-T_{i, j}^{0}}{1 / 2 \Delta t}= & \frac{k \Delta y \Delta z}{\Delta x}\left[\left(T_{i+1, j}^{*}-T_{i, j}^{*}\right)-\left(T_{i, j}^{*}-T_{i-1, j}^{*}\right)\right] \\
& +\frac{k \Delta x \Delta z}{\Delta y}\left[\left(T_{i, j+1}^{0}-T_{i, j}^{0}\right)-\left(T_{i, j}^{0}-T_{i, j-1}^{0}\right)\right],
\end{aligned}
$$

$$
\begin{aligned}
\rho c_{p} \Delta x \Delta y \Delta z \frac{T_{i, j}^{* *}-T_{i, j}^{*}}{1 / 2 \Delta t}= & \frac{k \Delta y \Delta z}{\Delta x}\left[\left(T_{i+1, j}^{*}-T_{i, j}^{*}\right)-\left(T_{i, j}^{*}-T_{i-1, j}^{*}\right)\right] \\
& +\frac{k \Delta x \Delta z}{\Delta y}\left[\left(T_{i, j+1}^{* *}-T_{i, j}^{* *}\right)-\left(T_{i, j}^{* *}-T_{i, j-1}^{* *}\right)\right],
\end{aligned}
$$

when applying Fourier's law of heat conduction and using the formalism of the ADI method. The numerical grid cell size is denoted $\Delta x \times \Delta y \times \Delta z$. The $x$-direction has - arbitrarily - been chosen to be the implicit direction in the first sub-timestep (where super-scripts 0 and ${ }^{*}$ mean explicit and implicit, respectively) and explicit in the second (where super-scripts * and ${ }^{* *}$ mean explicit and implicit, respectively). The inclusion of $\Delta z$ in Eqs. (6) and (7) is done in order to emphasize the importance of using the correct control volume when including the loss terms defined below in Eq. (12).

Eqs. (6) and (7) can be re-written in the form

$$
\begin{aligned}
& a_{i, j} T_{i, j}^{*}=b_{i, j} T_{i+1, j}^{*}+c_{i, j} T_{i-1, j}^{*}+d\left(T_{i, j}^{0} ; T_{i, j+1}^{0} ; T_{i, j-1}^{0}\right), \\
& a_{i, j} T_{i, j}^{* *}=b_{i, j} T_{i, j+1}^{* *}+c_{i, j} T_{i, j-1}^{* *}+d\left(T_{i, j}^{*} ; T_{i+1, j}^{*} ; T_{i-1, j}^{*}\right),
\end{aligned}
$$

where $a_{i, j}, b_{i, j}$ and $c_{i, j}$ are assumed constant throughout the timestep and $d$ includes the explicit and other additive terms. This function may be spatially dependent if, e.g. $c_{p}$ is. Solving Q1 Eqs. (8) and (9) is equivalent to inverting a tri-diagonal matrix. Such an inversion is linearly time-consuming in the number of mesh points and can be done using the well-known Tri-Diagonal Matrix Algorithm (TDMA) given in, e.g., Patankar (1980).

The coupling between the four domains is done using Fourier's law of heat conduction formulated through the use of thermal resistances (see the schematic in Fig. 2).

The heat capacity of the MCM is typically a strong function of both temperature and magnetic field. It is calculated from MFT and in this implementation a large dataset of $c_{\mathrm{p}}$ values has been tabulated with a sufficient range in both temperature and field. The value of $c_{p}$ needed for every sub-timestep is then spline-interpolated in this dataset using a natural cubic spline (Press et al., 1992).

The forced convection term, due to the fluid movement, is implemented following the "up-wind scheme" (see Patankar, 1980). This ensures that the thermal energy of the up-wind cell influences the convection term rather than using the centered difference.

The boundary conditions are given in Figs. 1 and 4. The initial condition was for all experiments set to be a uniform temperature of $298 \mathrm{~K}$ throughout the domains.

In Petersen et al. (2008) the original numerical model is thoroughly validated. The new implementation has been exposed to the same tests and is equally numerically valid and in some cases (especially when heat conservation is crucial) the new implementation is more accurate. 
Fig. 2 - The xy-plane of the replicating cell (half a fluid channel and half a plate of MCM and flow guides). The two boundaries marked "symmetry" are symmetric, or adiabatic, due to the nature of the representation using half a replicating cell. The internal boundaries are marked with their respective thermal resistances.

\subsection{The magnetocaloric effect as a source term}

Previously the change in magnetic field was modeled as an abrupt or discrete change as described in Petersen et al. (2008). This approach is obviously not physically correct, but may be sufficient to a certain extent. In order to improve this aspect of the modeling, the change in magnetic field could be implemented to happen through a number of timesteps. One approach would be a simple ramping of the field through an appropriate function, e.g. linear, sinosoidal or hyperbolic tangent. An even more realistic solution is to model the physical movement of the regenerator in and out of the magnetic field from a specific magnet configuration. If the field profile is known then the magnetic flux density as a function of time (and space) is trivial to derive.

Whichever method is used for modeling the magnetic field change in a continuous way, the MCE has to be formulated as a source term in the thermal equation for the MCM. Assuming adiabatic magnetization through each timestep, the heat energy released from the change in magnetic field from time $t$ to $t+\Delta t$ is calculated on basis of the known, or explicit,

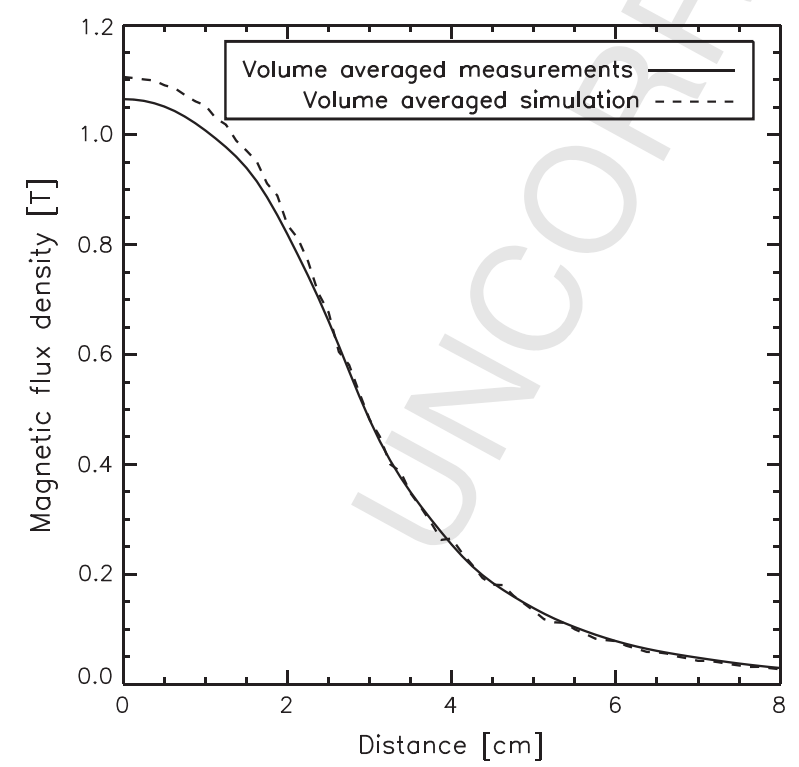

Fig. 3 - The profile of the magnetic flux density of the permanent Halbach magnet used in the experiments. Shown are both the measured data values and the corresponding model results. temperature (i.e. $\left.T_{i, j}(t)\right)$. The differential adiabatic temperature change can be written as

$\left.\frac{\mathrm{d} \Delta \mathrm{T}_{\mathrm{ad}}}{\mathrm{dt}}\right|_{\mathrm{t}}=-\left.\left.\mu_{0} \frac{\mathrm{T}(\mathrm{t}) \partial \mathrm{m}}{\mathrm{c}_{\mathrm{p}}(\mathrm{t}) \partial \mathrm{T}}\right|_{\mathrm{t}} \frac{\mathrm{dH}}{\mathrm{dt}}\right|_{\mathrm{t}}$.

Here indices $i, j$ have been omitted for simplicity and $\mu_{0}$ is the vacuum permeability, $m$ is the specific magnetization and $H$ is the magnetic field. Multiplying by $\Delta x \Delta y \Delta z \rho c_{p}$ on both sides of Eq. (10) the MCE source term $Q_{M C E}$ becomes

$Q_{\text {MCE }}=-\left.\left.\mu_{0} \rho T(t) \frac{\partial m}{\partial T}\right|_{t} \frac{d H}{d t}\right|_{t} \Delta x \Delta y \Delta z$.

Eq. (11) can be inserted directly on the right hand side of Eqs. (6) and (7) as a source term.

The temporal rate of change of the magnetic field $\mathrm{dH} / \mathrm{dt}$ is derived from the field profile of the used magnet system. In Fig. 3 the flux density of the permanent Halbach magnet system used in the AMR experiments is given as a function of distance from the centre of the bore of the Halbach cylinder. The figure shows both the measured flux density and modeling data using the model from Bjørk et al. (2008).

\subsection{The 2.5D heat loss formulation}

The experimental setup (Bahl et al., 2008) does not include heat exchangers but does of course leak heat to the surroundings. These are two major differences between the model and the experiment. It is expected that the performance in general will be over-estimated by the model since it is somewhat ideal without losses and that the trends in performance (both in load and no-load situations) will be reproduced fairly well by the model. This is due to the fact that the model actually resolves the important parts of the experimental geometry well and the geometrical parameters are expected to be crucial for the trends of a parallel-plate AMR device.

However, to improve the model, heat losses have been implemented as an alternative to the original HEXs modeled as copper plates. The heat loss is implemented through a lumped analysis and under the assumption that the replicating cell under consideration looses most of its heat in the not-resolved z-direction. The loss can then be implemented as an additional term in Eqs. (6) and (7) using the formalism of thermal resistance: 


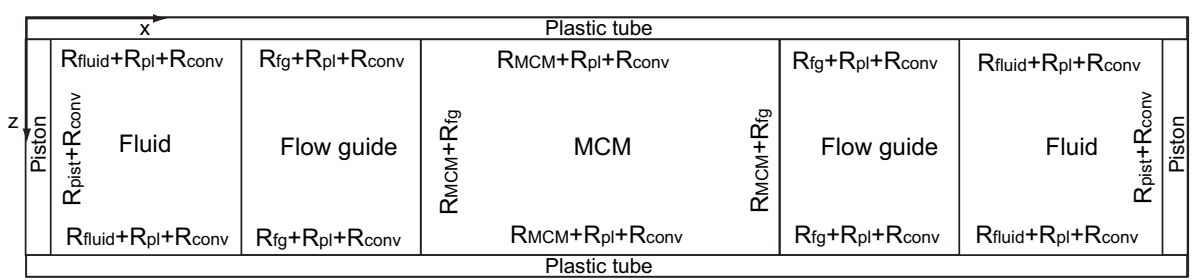

Fig. 4 - The $x z$-plane of the system. The z-direction is not resolved, however, the 2.5D model takes the distance from the centre of the control volumes to the ambient into account when calculating the thermal losses, as indicated in the figure.

$\mathrm{Q}_{\text {loss }}=\frac{\mathrm{T}_{\infty}-\mathrm{T}_{\mathrm{i}, \mathrm{j}}}{\sum_{l} \mathrm{R}_{l}}$

where the total thermal resistance from the centre of the cell (in terms of the $\mathrm{z}$-direction) to the ambient is denoted by $\sum R_{l}$. There are three terms in this sum. First the thermal resistance through the material within the regenerator $R_{\mathrm{m}}$ (fluid or solid). Second, the housing of the regenerator block $R_{\mathrm{pl}}$ (made of a plastic material) and finally loss via natural convection to the ambient $R_{\text {conv }}$

$\sum_{l} R_{l}=R_{m}+R_{\mathrm{pl}}+R_{\text {conv }}=\frac{1 / 2 \Delta z}{k_{m} \Delta x \Delta y}+\frac{1 / 2 \Delta z}{k_{\mathrm{pl}} \Delta x \Delta y}+\frac{1}{h_{\text {conv }} \Delta x \Delta y}$

This 2.5D thermal loss formulation is schematically visualized in Fig. 4. The loss to the ambient through natural convection is characterized by the parameter $h_{\text {conv }}$. Textbook values suggest that $h_{\text {conv }}$ lies in the range $5-20 \mathrm{~W} / \mathrm{K} \mathrm{m}^{2}$ (Holman, 1987). The thermal properties of the plastic housing are given in Table 1.

\section{Results and discussion}

In this section the numerical model is compared to various experiments performed with the experimental AMR device located at Ris $\varnothing$ DTU (Bahl et al., 2008). The model is able to operate in different configurations (2D ideal with no thermal losses, 2.5D with thermal losses, discrete or continuous magnetic field, etc.). Therefore various situations have been picked out for investigation. First, in Section 3.1 variation of the fluid displacement is investigated and compared to the experimental and original model data. Second, in Section 3.2 variation in the timing of the AMR cycle is explored. Third, in Section 3.3 the variation in the application of the magnetic field is compared between the ideal model, the 2.5D loss model and the experiment.

Table 1 - Values of the various thermal properties of the materials used

\begin{tabular}{lccc} 
Material/property & $k[\mathrm{~W} / \mathrm{m} \mathrm{K}]$ & $\rho\left[\mathrm{kg} / \mathrm{m}^{3}\right]$ & $c_{\mathrm{p}}[\mathrm{J} / \mathrm{kg} \mathrm{K}]$ \\
\hline Copper & 401 & 8933 & 385 \\
Water & 0.595 & 997 & 4183 \\
Gadolinium & 10.5 & 7900 & $\begin{array}{c}\text { 170-300 (temperature } \\
\text { and field dependent) }\end{array}$ \\
Plastic & 0.2 & 800 & 1250
\end{tabular}

Table 2 gives the specifications of each experiment/model. All experiments were carried out with $0.9 \mathrm{~mm}$ thick plates of pure Gd (obtained from China Rare Earth Materials Co.) with a spacing of $0.8 \mathrm{~mm}$. The experiments were all equipped with 13 plates. For further details on the experimental setup see Bahl et al. (2008).

\subsection{Fluid displacement experiments}

The fluid displacement, $\delta x$, is one of the key process parameters for an AMR. In Bahl et al. (2008) the dependency of the AMR performance on this parameter is studied using the experimental device and a slightly changed version of the numerical model of Petersen et al. (2008). However, the model did not include losses in the z-direction and the plastic flow guides were lumped to represent the entire loss of the plastic tube and regenerator housing. The geometric and operational parameters in the $2.5 \mathrm{D}$ loss model were set to resemble the configuration of the original experiment and to use MFT for modeling the MCE. The results are seen in Fig. 5.

The directly measured adiabatic temperature change of the Gd plates when using the Halbach magnet assembly is taken from Bahl and Nielsen (2008). A new experiment series was performed varying the fluid displacement. The model was adjusted to use MFT for calculating the MCE and also to use the directly measured $\Delta T_{\text {ad }}$ values. The heat capacity was in both cases determined from MFT. The results are seen in Fig. 6.

The fluid displacement experiments show a clearly asymmetric bell-shaped curve (Figs. 5 and 6). This shape is reproduced fairly well by the model. The peak in the curve is situated at a fluid movement around $40 \%$. There is

Table 2 - An overview of the experiments conducted in this work. The process parameters (fluid movement, timing and magnet assembly) are presented. The parameters apply both for the experiment and the corresponding modeling

\begin{tabular}{lcccl} 
Model & $\delta \mathrm{x} \%$ & $\tau_{\text {tot }}[\mathrm{s}]$ & \multicolumn{1}{c}{$\tau_{\text {rel }}$} & Magnet \\
\hline Stroke, (Bahl et al., 2008) & $5-95$ & 12 & 1 & Electro \\
Stroke, new experiments & $5-95$ & 8.2 & 0.51 & Halbach \\
Timing, (Bahl et al., 2008) & 50 & $12-18$ & $0.25-4.5$ & Electro \\
Timing, new experiments & 50 & 9 & $0.25-3.0$ & Halbach \\
Varying magnetic & 40 & 11.8 & 1.03 & Halbach \\
$\quad$ flux density & & & &
\end{tabular}




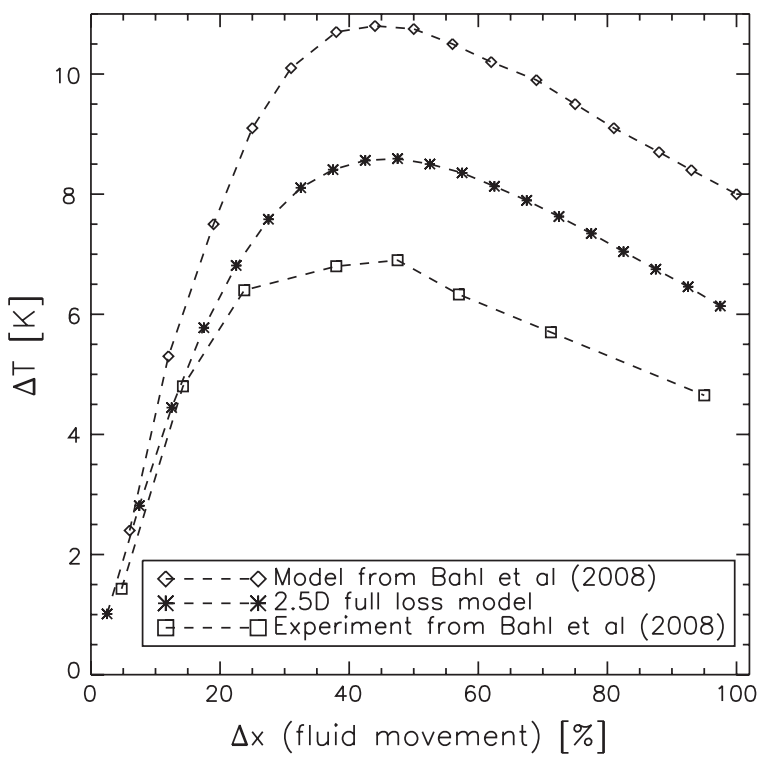

Fig. 5 - Fluid movement experiment from Bahl et al. (2008) with modeling results from both the original paper and this work. The model from this work used MFT to calculate the MCE, since it is not possible to translate the measured $\Delta T_{\text {ad }}$ values to a different magnetic field profile (the original experiment used an electromagnet).

a significant improvement when the experimentally determined values for the adiabatic temperature change are used instead of the mean field model.

\subsection{Timing experiments}

The timing of the AMR cycle is important for the performance of the system. There are two critical questions that need

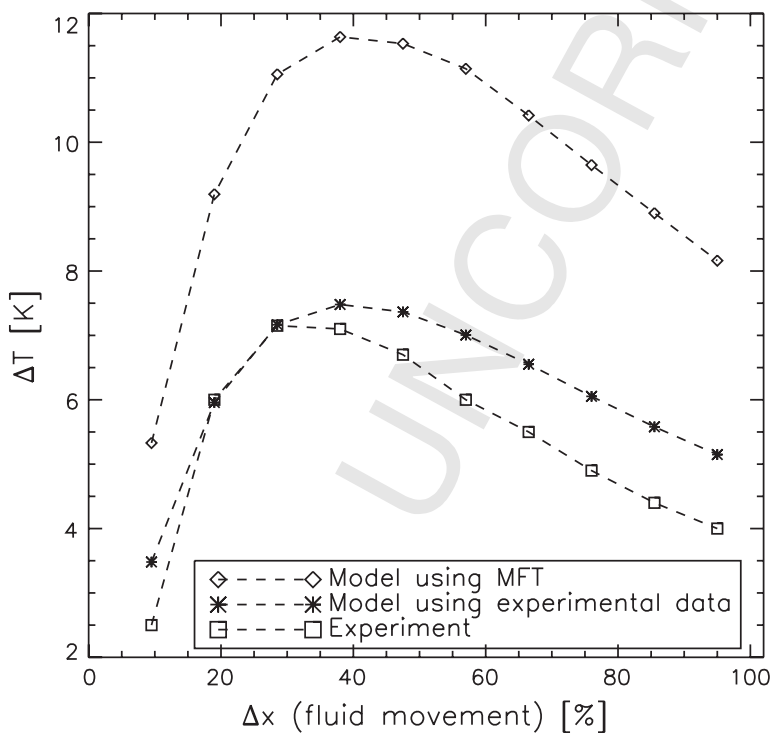

Fig. 6 - A new fluid movement experiment performed using the permanent Halbach magnet and modeled both using MFT and the measured adiabatic temperature changes.

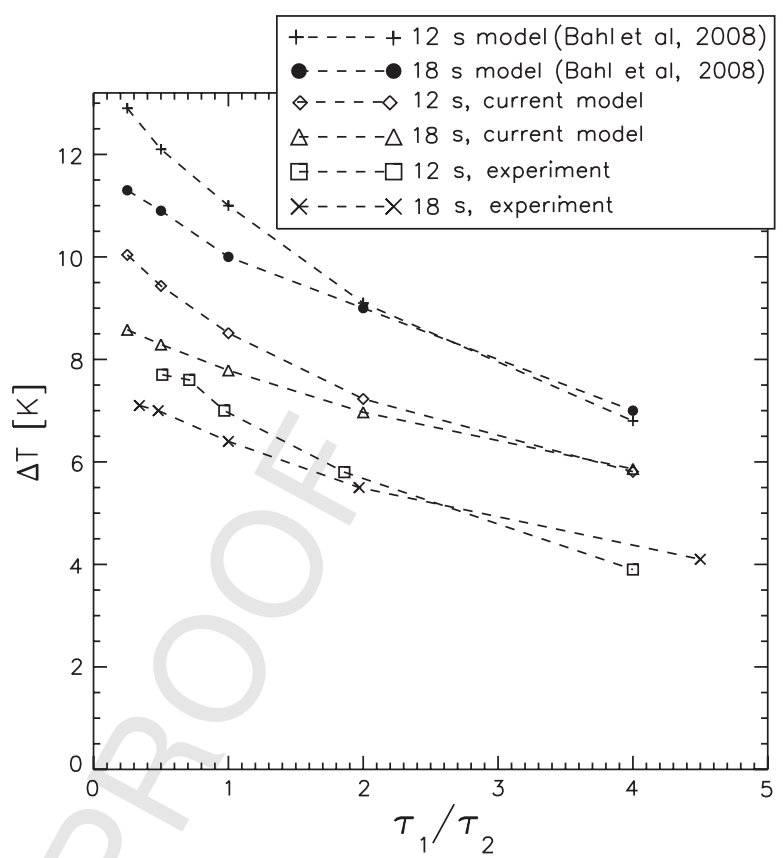

Fig. 7 - Timing experiment and corresponding modeling from Bahl et al. (2008) with the MFT-based 2.5D loss model from this work overplotted.

answering. First of all how long the entire cycle $\left(\tau_{\text {tot }}\right)$ should be. Secondly, the amount of time used for magnetizing/demagnetizing and afterwards reaching thermal equilibrium $\left(\tau_{1}\right.$ and $\tau_{3}$ ) compared to the time spent moving the fluid during the blow periods $\left(\tau_{2}\right.$ and $\left.\tau_{4}\right)$. In Bahl et al. (2008) experimental and model results are given for AMR setups where $\tau_{\text {rel }}$ and $\tau_{\text {tot }}$ are varied. The $2.5 \mathrm{D}$ loss model was setup to the same geometrical

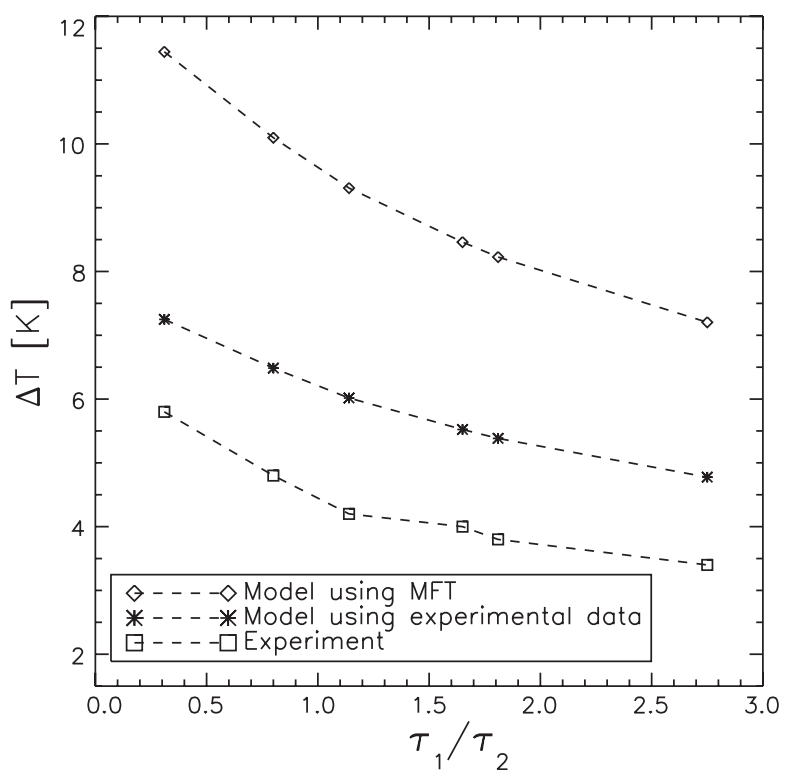

Fig. 8 - New timing experiment performed using the permanent Halbach magnet. The corresponding modeling has been performed for two cases, one using MFT and one using the measured $\Delta T_{a d}$ values. Both were done using the 2.5D loss formulation. 
and process parametric configuration. The results are given in Fig. 7.

A new experiment series, again using the Halbach magnet was performed varying $\tau_{\text {rel }}$ and the input parameters to the 2.5D loss model were set accordingly. Both the MFT and the directly measured $\Delta T_{\text {ad }}$ were used for modeling the MCE. The results are given in Fig. 8 .

The timing experiments, presented in Figs. 7 and 8, show that the no-load temperature span decreases as a function of the fraction $\tau_{\text {rel }}$. This behaviour is also well reproduced by all the models. In Fig. 7 a cross-over is seen between the curves for $\tau_{\text {tot }}=12$ and $18 \mathrm{~s}$ at large $\tau_{\text {rel }}$. This is also a feature that the models reproduce.

However, all the models using MFT for calculating $\Delta T_{\text {ad }}$ over-estimate the temperature span significantly. Using the measured $\Delta T_{\mathrm{ad}}$ values the model is seen to reproduce the absolute temperature spans to a higher degree. This is the same conclusion as for the fluid displacement experiments.

\subsection{Varying the magnetic flux density}

The magnetic flux density of the Halbach cylinder as a function of distance from the centre of the bore is given in Fig. 3. An experiment has been conducted where the regenerator was moved from the centre of the Halbach magnet out to various distances in an otherwise identical experiment. It is seen from the results in Fig. 9 that at a certain distance (approximately $7 \mathrm{~cm}$ ) the magnetic flux density is low enough that moving the regenerator further out does not increase the temperature span.

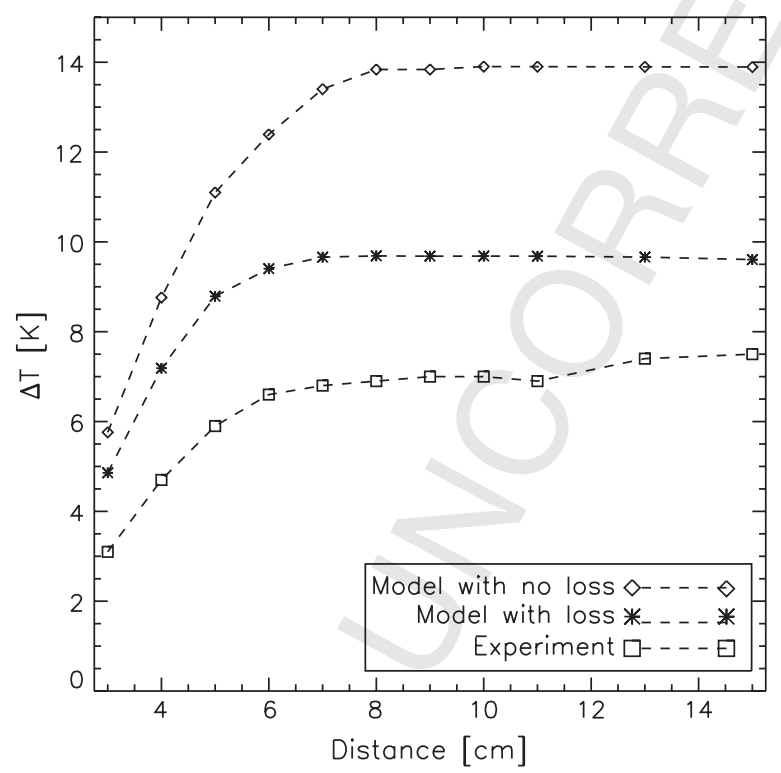

Fig. 9 - Experiment performed by altering the distance from the centre of the magnet bore that the regenerator is moved out to. Two modeling cases are seen. One with the ideal (not including 2.5D losses) and one with the 2.5D loss formulation. The trends are clearly seen to be reproduced, though the absolute values are not quite the same in the models as in the experiment.
The modeling of the varying magnetic flux density was done in two ways, both using the MCE described as a source term (see Subsection 2.3). One model-series was performed with the ideal (no heat loss) setup and the other with the 2.5D loss formulation. The results are shown in Fig. 9.

The experimental and modeling investigations of the sensitivity towards the change in field result in two interesting conclusions. First, the need for moving the regenerator far out of the field is limited to roughly $7 \mathrm{~cm}$ for the present system configuration. This fact is important. The timing of the experiment is to a certain degree dependent on how much time is spent moving the regenerator in and out of field. As seen from the timing experiments the time spent during this process should be as short as possible. Second, in Fig. 9 it is seen that including thermal parasitic losses in the model significantly improves the results of the model compared to the experiment. In both cases the MFT was used to model the MCE. Thus, only the inclusion of thermal parasitic losses can explain the clear improvement of the modeling results. It should be emphasized that the remaining difference between the model and the experiments is probably due to the additional heat losses in the experimental setup which have not been included in the model due to the lack of knowledge for the origin of these losses.

\subsection{Overall discussion}

The three different no heat-load situations experimentally investigated and numerically modeled here all point in the same direction. When keeping all parameters except one fixed the tendency in no-load temperature span is well described by the models. However, there is a tendency for the models to over-estimate the absolute values of the temperature span. The reason for this is primarily that the MFT is too idealized and that real experimental data should be used instead. This is supported by the results from the modeling when using the measured values of the adiabatic temperature change.

Furthermore, passive regeneration in the plastic housing may be significant for the performance of the AMR. Generally, the thermal losses to the ambient may be more tightly dependent temporarily (through the cycle). This cannot be investigated in the present model and full three-dimensional modeling is needed to investigate this.

\section{Conclusion}

A re-definition, re-implementation and feature-upgrade of the numerical 2D AMR model (Petersen et al., 2008) were presented. The computation time has been reduced by a factor of 100. This allows for large parameter space surveys which are under preparation for future publication.

The current state of the 2D AMR model has been investigated and presented. It is concluded that the 2.5D loss model is a significant improvement in terms of reproducing the experimental results. The continuous description of the change in magnetic flux density is recognized as an important improvement of the model in terms of operating the experiment and confidence that the model is well-represented using the discrete change if needed. 
Including the measured adiabatic temperature change in the experimental setup with the Halbach magnet has enabled the model to reproduce all aspects of the no heat-load experiments reasonably well.

On the basis of the results presented in this paper it is concluded that the ideal 2D model can be used to explore the performance of a linear reciprocating parallel-plate based AMR design. Once the optimal configuration settings have been found, the 2.5D full loss model can be used to explore the expected experimental performance in more detail. The reason for not only using the loss model is that the ideal AMR work is independent of experimental shortcomings and choices. The results from such an ideal AMR study can thus be used by other experiments and provide a more general understanding of the details and theory of AMR.

\section{Acknowledgements}

The authors thank Jørgen Geyti for technical assistance. Furthermore the authors would like to acknowledge the support of the Programme Commission on Energy and Environment (EnMi) (Contract no. 2104-06-0032) which is part of the Danish Council for Strategic Research.

\section{R E F E R E N C E S}

Allab, F., Kedous-Lebouc, A., Fournier, J., Yonnet, J., 2005. Numerical modeling for active magnetic regenerative refrigeration. IEEE Transactions on Magnetics 41 (10), 37573759.

Bahl, C., Nielsen, K., 2008. The effect of demagnetization on the magnetocaloric properties of gadolinium. Journal of Applied Physics 105, 013916.

Bahl, C., Petersen, T., Pryds, N., Smith, A., 2008. A versatile magnetic refrigeration test device. Review of Scientific Instruments 79 (9), 093906.

Bjørk, R., Bahl, C.R.H., Smith, A., Pryds, N., 2008. Optimization and improvement of Halbach cylinder design. Journal of Applied Physics 104 (1), 013910.

Comsol, 2005. In: Comsol Multiphysics Model Library, third ed. COMSOL AB, Chalmers Teknikpark $41288 \mathrm{G}$.
Dikeos, J., Rowe, A., Tura, A., 2006. Numerical analysis of an active magnetic regenerator (amr) refrigeration cycle. AIP Conference Proceedings 823 (1), 993-1000.

Engelbrecht, K., Nellis, G., Klein, S., Boeder, A., 2005. Modeling active magnetic regenerative refrigeration systems. In: Egolf, P.W. (Ed.), First International Conference on Magnetic Refrigeration at Room Temperature. Refrigeration Science and Technology Proceedings, vol. 1, pp. 265-274.

Fox, R.W., McDonald, A.T., 1994. Introduction to Fluid Mechanics. John Wiley and Sons, Inc.

Gao, Q., Yu, B., Wang, C., Zhang, B., Yang, D., Zhang, Y., 2006. Experimental investigation on refrigeration performance of a reciprocating active magnetic regenerator of room temperature magnetic refrigeration. International Journal of Refrigeration 29, 1274-1285.

Holman, J., 1987. In: Heat Transfer, first ed. McGraw-Hill.

Lautrup, B., 2005. Physics of continuous matter. Exotic and Everyday Phenomena in the Macroscopic World. Institute of Physics.

Li, P., Gong, M., Yao, G., Wu, J., 2006. A practical model for analysis of active magnetic regenerative refrigerators for room temperature applications. International Journal of Refrigeration 29, 1259-1266.

Okamura, T., Yamada, K., Hirano, N., Nagaya, S., 2006. Performance of a room-temperature rotary magnetic refrigerator. International Journal of Refrigeration 29, 1327-1331.

Patankar, S.V., 1980. Numerical Heat Transfer and Fluid Flow. Taylor \& Francis.

Pecharsky, V.K., Gschneidner, K., 2006. Advanced magnetocaloric materials: what does the future hold? International Journal of Refrigeration 29, 1239-1249.

Petersen, T.F., Pryds, N., Smith, A., Hattel, J., Schmidt, H., Knudsen, H., 2008. Two-dimensional mathematical model of a reciprocating room-temperature active magnetic regenerator. International Journal of Refrigeration 31, 432-443.

Press, W.H., Teukolsky, S.A., Vetterling, W.T., Flannery, B.P., 1992. Numerical recipes in FORTRAN. In: The Art of Scientific Computing, second ed. University Press, Cambridge.

Rowe, A., Barclay, J., 2003. Ideal magnetocaloric effect for active magnetic regenerators. Journal of Applied Physics 93 (3), 16721676.

Rowe, A., Tura, A., 2008. Active magnetic regenerator performance enhancement using passive magnetic materials. Journal of Magnetism and Magnetic Materials 320 (7), 1357-1363.

Rowe, A., Tura, A., Richard, M.-A., Chahine, R., Barclay, J., 2004. Magnetic refrigeration - an overview of operating experience using the amr test apparatus. Advances in Cryogenic Engineering 49 (B), 1721.

Shir, F., Mavriplis, C., Benneth, L., Torre, E., 2005. Analysis of room temperature magnetic regenerative refrigeration. International Journal of Refrigeration 28, 616-627. 